\title{
PROYECTOS DEPORTIVOS SOCIALES: UN ANÁLISIS HISTÓRICO DE SU SURGIMIENTO Y DESARROLLO EN LA CIUDAD DEL RIO DE JANEIRO
}

Tibério Machado 1,2,3 y Angelo Vargas 2,3,4

' Docente do Curso de Educação Física - UNIG

${ }^{2}$ Laboratório de Estudos da Cultura Social Urbana - LECSU

3 Grupo de Estudos em Direito Esportivo - FND/UFRJ

4 Docente da Faculdade Nacional de Direito - UFRJ

tiberiojose@gmail.com y angelo.vargas@uol.com.br

RESUMEN:

Durante el proceso de desarrollo humano, la relación de interacción entre el hombre y el deporte se convirtió indiscutible. El deporte, además de compañero atendió diversos anhelos humanos, actuando como una estrategia para entrenamiento de las habilidades sociales, especialmente en el universo de los proyectos deportivos sociales. El objetivo del presente estudio fue por medio de la investigación documental investigar el proceso de desarrollo de los proyectos deportivos sociales en la ciudad de Rio de Janeiro, sobre todo con el apoyo del gobierno local. Los resultados favorecen la compresión de que este tipo de acción, que tiene como objetivo la difusión de preceptos positivos a través del desenvolvimiento deportivo, presentó crecimiento y fomentación indudable, sobre todo después de la participación gubernamental, pero su surgimiento no presentó el apoyo del gobierno. Los objetivos deseados en los proyecto administrados por la Secretaria Municipal de Deporte y Ocio de la ciudad del Rio de Janeiro, favorecen la comprensión de que muchos objetivos son deseados. Siendo así, fue posible concluir que iniciativas análogas, deben ser estimuladas en todos los sectores de la sociedad, especialmente con el fin de ofrecer todos los beneficios envueltos en el deporte y así contribuir para la formación de ciudadanos.

PALABRAS CLAVE: Políticas Públicas, Deporte y Vulnerabilidad Social. 


\section{INTRODUCCIÓN.}

A lo largo de la historia, se convirtió indiscutible la relación entre el Hombre y la realización de actividades corporales, que en el campo semántico deportivo no sólo se resume al universo de los juegos y competiciones, pero abarcó una amplia gama de objetivos, en especial con respecto a las necesidades presentadas por la población en la sociedad contemporánea.

Con objetivo de proporcionar el entrenamiento de las habilidades sociales, el deporte en la representación de la práctica deportiva hay ganado visibilidad y espacio, caracterizado por el atendimiento por prioridad de la población pobre, que demostró como peculiaridad la necesidad de ayuda o asistencia social. El deporte en la sociedad contemporánea emergió con la capacidad de proporcionar situaciones en el desarrollo de las actividades deportivas, que son favorables para la promoción y diseminación de preceptos de valor positivo y fundamental para la vida en sociedad (Tubino, 2001).

\section{1.- Relación hombre y el deporte: un bosquejo histórico}

Una delimitación sobre en principio de la relación entre el Hombre y la práctica deportiva o deporte es difícil, debido las diferentes formas de su presentación en la sociedad, sin embargo, la literatura permitió identificar que esto consolidado fenómeno social nombrado deporte, hay acompañado los seres humanos durante todo el proceso de la evolución (Rubio, 2002; Machado \& Vargas, 2011).

En la prehistoria, lo que podría ser denominado deporte, presentó característica guerrera, ritualista y utilitaria. Sin embargo, es mister asegurar que en este período, la práctica deportiva presentaba la ausencia de reglas y normalizaciones, fundamentalmente porque los ejercicios desarrollados tenían finalidad de supervivencia, como la caza y la pesca (Rubio, 2002).

No obstante, el deporte emergió en la Antigua Grecia segundo Lefèvre (2003,) con una significativa representación cultural, tiendo como acontecimiento principal los Juegos Griegos, que pueden considerarse la génesis de los Juegos Olímpicos de los tiempos modernos. Es importante tener en cuenta que el pueblo griego tenía una óptica y un pensamiento peculiar, donde lo cuerpo no fue asociado solamente a un símbolo de belleza, pero fue comprendido como una representación de sabiduría y el pensamiento humano, hecho presente y presentado en la "Máxima de Juvenal": "Mens sana in corpore sano" (Castellani Filho, 1988, p. 48).

Los grandes espectáculos deportivos y culturales fueron las características primordiales presentadas por el deporte en la Antigua Roma, donde en objetivo era ofrecer diversión y entretenimiento para la población. Sin embargo, un análisis en mayor profundidad permitió la comprensión de que todo ese escenario de diversión presentado, en verdad representa otra realidad, que constituye una estrategia política de cooptación de los espectadores. Siqueira (2006) infiere que: "o alimento e o entretenimento que deveriam ser dados ao povo para que se mantivesse submisso" (p. 87). La naturaleza política asumida por el deporte se hizo famosa, sobre todo por los incentivos y estímulos recibidos, que fue registrado en la historia del mundo como "Panis et circense" (Saba, 2001).

Cuanto al Periodo Medieval, el deporte se caracterizó por la oscuridad, en lo que respecta a el desarrollo de los juegos como una forma de entretenimiento e identidad popular. El Clero promovió la elitización del deporte, utilizando de las prácticas 
recreativas y deportivas para su diversión y entretenimiento. Sin embargo, el pueblo o la "plebe" no fueron incluidos en este privilegio, no siendo posible su participación y teniendo como alternativa solamente la observación (Tubino, 2005; Golf \& Truong, 2006).

Destarte, un momento en la historia humana que representó un significativo avance para el desarrollo deportivo, respecta al Post Segunda Guerra Mundial. En esta época, hubo un importante desenvolvimiento y crecimiento de los deportes, siendo alentados debido a la disputa entre los países que formaran el "Bloque Socialista y el "Bloque Capitalista". Este hecho, corroboró considerablemente para al avance tecnológico y el crecimiento de las políticas deportivas en el mundo (Tubino, 2005), empero los objetivos reales se basaron en la demostración de fuerza y superioridad a través del deporte, hecho que ayudó para lo mantenimiento de la disputa entre los dos bloques.

En el siglo XIX, los deportes modernos emergieron en la Inglaterra, debido la influencia de Thomas Arnold, que estimuló la creación de reglamentos específicos para las modalidades deportivas, con el objetivo de fomentar el deporte (Tubino, 1993). Tiendo como base conceptual los postulados filosóficos de Rousseau, el deporte en este contexto presentó como característica la relación entre en ejercicio físico y el pensamiento humano. Esta nueva dimensión promovió una influencia notable en los ideales del humanista francés Pierre de Coubertain, que emergió en la historia deportiva del mundo como la personalidad responsable por la resurrección de los Juegos Olímpicos (Rosseau, 1995).

Cabe señalar que en este período, el deporte emergió con el objetivo pacificador y unificador, siendo entendido como una estrategia para promover la paz. Durante la realización de los Juegos Olímpicos, ocurrió un momento de tregua en las batallas, tiempo nombrado y conocido en la historia del mundo como "Tregua Olímpica". Además, tales competiciones simbolizaran una forma de unir las diversas naciones en el mismo evento (Tubino, 1993; Vargas, 2006).

En la sociedad moderna, primordialmente en la República Federativa del Brasil, el deporte se ha caracterizado como una estrategia importante para el entrenamiento de habilidades sociales, sobre todo con el objetivo de atendimiento de las necesidades de los sectores desfavorecidos de la población, siendo una alternativa con finalidad y característica política. (Zaluar, 1994). Esta perspectiva del deporte fue discutida por Tubino (2001), donde el autor infiere acerca de la importancia del deporte en la formación de los niños y jóvenes pobres:

O esporte é como um instrumento fundamental no auxílio ao processo de desenvolvimento integral das crianças, dos adolescentes e dos jovens; respeitando as experiências e expectativas individuais, democratizando o acesso a espaço esportivo, valorizando o esporte como complementar a técnica de saúde preventiva, incutindo valores éticos e sociais, resgatando a cultura esportiva (pp. 10).

Mediante la valorización del deporte y el crecimiento de los problemas sociales en la sociedad brasileña, la actuación del Gobierno se hizo activa después de la promulgación del Artículo 217 de la Constitución de la República Federativa del Brasil, en 5 de octubre de 1988, que estableció en siguiente texto: "É dever do Estado fomentar as práticas desportivas formais e não formais como direito de cada um..." (Brasil, 1988). No obstante, la diseminación deportiva por el poder público se ha convertido en indiscutible y evidente cuando se analiza el contexto de los proyectos deportivos sociales, que presentaron entre sus ideales y metas promover una 
alternativa de socialización para los residentes de localidades con bajo recurso y quienes en su mayoría viven en situación de exclusión, degradación y segregación social (Zaluar, 1994; Vargas, 2002).

\section{2.- La sociedad contemporánea brasileña: un contexto con diversos problemas sociales}

La sociedad contemporánea brasileña presenta peculiaridades y características que pueden ser clasificadas como socialmente degradantes. Su contexto social fue clasificado como de riesgo social, debido a los problemas de su ambiente y los efectos nocivos que pueden causar en los individuos envueltos. De acuerdo con Malta, Veras e Vasconcelos (2008) las situaciones que denotan riesgo social deben ser entendidas a través de: "verdadeiras causas que levam os meninos e meninas às ruas ou a situações de violência, de pobreza material e social." (p. 296).

El contexto social con característica degradante terminó perjudicando el desenvolvimiento de los jóvenes, que están en un proceso de formación y creación de una identidad social, además de su mayor vulnerabilidad, mostraron una incidencia significativa de la participación de las situaciones enumeradas (Vargas, 2002). Los postulados filosóficos de Marx y Engels (1999) nos permiten comprender que el Hombre emergió como un producto del ambiente donde se inserta y están expuestos a todas las intervenciones presentes en el contexto social y que pueden reflejar o no. De la misma manera Vargas (2002) corroboró con el argumento que los más jóvenes tienen mayor probabilidad de reproducción de los valores, gestos y acciones presenciado en su vida cotidiana y el ambiente de convivencia, presentando como característica la motricidad con estereotipo degradante.

Sin embargo, las características de un contexto social pueden tener relación con el nivel de las acciones y recursos emprendidos por el gobierno, principalmente en la forma de servicios sociales. La ausencia o insuficiencia de actuación gubernamental pueden proporcionar un vacío, que puede favorecer el desarrollo de las acciones de magnitud nocivas para la población, contribuyendo para el desenvolvimiento de las características de riesgo social, hecho que es favorable para la formación del escenario de degradación. (Grostein, 2001). La presencia de la violencia y de la delincuencia son características que confirman el contexto de riesgo social, además de emerger como circunstancias que tienden a crecer en el escenario que carece de asistencia social y hacen como la víctima principal los jóvenes (Vargas, 2002).

Hay que destacar que tales lugares, donde el contexto social expresa característica degradante, por consiguiente tienen baja clasificación en las investigaciones realizadas por el Índice de Desarrollo Humano (IDH) y el Índice de Desarrollo Social (IDS). Ambas clasificaciones, utilizan como parámetros indicadores sociales que tienen como objetivo evaluar la calidad de los servicios básicos disponibles en una determinada región, así como las particularidades sociales y educacionales (PROGRAMA DAS NAÇÕES UNIDAS PARA O DESENVOLVIMENTO (PNUD), 2001; CAVALLIERI; LOPES, 2008). Preponderantemente, las localidades con bajos índices, pueden expresar como características señales de desorden habitacional o como conocido popularmente en la República Federativa del Brasil "favelização", que se refiere al hecho de que la falta de actuación del Gobierno, no que respecta al desenvolvimiento de una política de habitación.

\section{3.- Proyecto deportivo social: una forma de fomentación deportiva por parte del gobierno brasileño}


Los proyectos deportivos sociales deben ser comprendidos como modelo de proyecto intervencionista, cuyo la importancia es propiciar el logro de los objetivos relacionados con el ámbito social, teniendo como peculiaridad principal la implementación del deporte como estrategia para el entrenamiento de las habilidades sociales. Cabe señalar, que el Artículo 217 de la Constitución de la República Federativa del Brasil, de 1988, consagra el mérito de la promoción de la educación través de la práctica deportiva. Además, el presente artículo infiere acerca de la obligatoriedad de la diseminación deportiva formal y no formal para los brasileños (Brasil, 1988). Tiendo su origen en las acciones sociales, que emergieron en la sociedad brasileña en los años 70 , los proyectos deportivos sociales han surgido, sobre todo, debido el crecimiento de los problemas sociales en la sociedad contemporánea (Zaluar, 1994).

En este universo, los años 80 emergió como un período de significativo crecimiento y fomentación de la práctica deportiva, primordialmente de las políticas públicas asociadas con el deporte. Como declarado por Zaluar (1994): "um projeto mais ambicioso que não se restringia à educação esportiva, mas que a utilizava como meio de educar crianças e jovens..." (p. 35). Es mister, la comprensión de que el Gobierno Brasileño entendió la necesidad de fomentación del deporte como estrategia de transformación y emancipación social, como presentado en el Artículo 217 de la Constitución de la República Federativa del Brasil, de 1988 y afirma el Baluarte de la Educación Física brasileña Manoel Tubino, que presentó una "nueva imagen" para el deporte. De acuerdo el autor, el deporte es un instrumento de colaboración para el desarrollo de los niños, los adolescentes y los jóvenes, lo que permite la difusión de los valores éticos que contribuyen para la formación humana y la vida en sociedad (Tubino, 2001).

Destarte, la importancia de demostrar la interacción que ocurrió entre las políticas públicas y el deporte en los años 80 , un hecho expresa una significativa relevancia. De acuerdo con el Artículo 217 de la Constitución de la República Federativa del Brasil, de 1988: "É dever do Estado fomentar práticas desportivas formais e não formais, como direito de cada um". En función de la dicha determinación, el desarrollo del deporte brasileño en todo el territorio nacional, se convirtió en obligatorio, siendo incumbencia del Estado Brasileño proporcionar un medio para su diseminación, ya que la práctica deportiva ahora hay recibido status de derecho social indubitable (Brasil, 1988).

Con respecto a la ciudad de Rio de Janeiro, durante los años 80, emergió un modelo de proyecto deportivo social nombrado villa olímpica, que ofrecía una diversidad de modalidades deportivas, con el objetivo de promover beneficios sociales, sobre todo en ofrecer una alternativa para la población pobre. Además, algunas unidades han proporcionado la oferta de servicios con finales sociales y que no tienen relación directa con la práctica deportiva, hay que son fomentados con el objetivo de promover la asistencia social de los ciudadanos de la región. (Machado, Dória \& Vargas, 2011).

Hoy en la sociedad brasileña la política deportiva social, en el universo de los proyectos deportivos sociales nombrados villas olímpicas, demuestra significante crecimiento y fomento por el Gobierno, sirviendo a los intereses y necesidades de la población. Por lo tanto, el presente estudio tiene como objetivo investigar el proceso histórico de surgimiento y implementación de los respectivos proyectos en la ciudad del Rio de Janeiro, además de presentar los objetivos que se persiguen al largo de los años y analizar cuál es la participación del Gobierno Brasileño en el desarrollo de este tipo de proyecto deportivo social. 


\section{METODOLOGÍA}

El presente estudio empleó dos instrumentos metodológicos diferentes, a fin de garantizar la fiabilidad y la profundidad en la investigación. Entre los instrumentos metodológicos, se usó el método de investigación conocido como Investigación Documental, que según Rampazzo (2005) ha caracterizado a la procura de documentos de fuentes primarias, que pueden ser archivos, fuentes estadísticas y fuentes no escritas. Este tipo de investigación ha permitido el uso de fuentes ricas y estables de datos, que son los documentos. Además de la investigación documental, este estudio utilizó el instrumento metodológico nombrado Estudio Histórico, que de acuerdo con Flegner (1995) el dijo recurso metodológico tiene como objetivo presentar la historia de forma continua, respetando la secuencia cronológica de los hechos ocurridos.

\section{RESULTADOS}

Los resultados permitieron la presentación cronológica de los acontecimientos de relevancia significante para el surgimiento y desarrollo de los proyectos deportivos sociales en la ciudad del Rio de Janeiro, administrado por la Prefectura local, nombrados como Villa Olímpica. Las informaciones presentadas fueran encontradas en la Constituicion de la Republica Federativa del Brasil, promulgada en 1988, en lo Manual de las Villas Olímpicas de la Ciudad del Rio de Janeiro, juntamente con los documentos y informaciones de los sitios web de la Prefectura de la Ciudad del Rio de Janeiro y de la Secretaria Municipal de Deporte y Ocio.

\section{1.- Presentación cronológica de los acontecimientos significantes para el crecimiento de los proyectos deportivos sociales nombrados villa olímpica en la ciudad del Rio de Janeiro}

Año de 1985: Creación de la Secretaria Municipal de Deporte y Ocio, en lengua portuguesa, Secretaria Municipal de Esporte e Lazer (SMEL), que hasta hoy mantiene su nombre y su efectividad en la gestión y fomentación del deporte y el ocio en la ciudad del Rio de Janeiro. Sin embargo, en este momento histórico, la respectiva secretaria se limitaba al desenvolvimiento de las acciones sociales, que eran programas de intervención específica, que presentaran poca profundidad o menos objetivos deseados, en lo que respecta a la asistencia de la población pobre. Además, las acciones sociales presentaran una pequeña actuación y pequeña duración.

Año de 1987: Surgimiento del primero proyecto deportivo social, con característica de una villa olímpica. Las instalaciones de la Villa Olímpica de la Mangueira se encuentran en el área norte de la ciudad, a cerca de una comunidad pobre nombrada "Morro da Mangueira". Este proyecto surgió como resultado de la asociación entre el "Grêmio Recreativo Escola de Samba Estação Primeira de Mangueira" y el Tercer Sector, teniendo esta iniciativa sustancial importancia y evidencia en los medios de comunicación, sirviendo como modelo para futuros proyectos análogos. Como característica principal, es posible citar la ausencia de participación del Gobierno, además de la asistencia de los jóvenes como una prioridad, sobre todo en lo que respecta a la ocupación del tiempo libre y por lo tanto la reducción de la ociosidad.

Año de 1988: Promulgación del Artículo 217 de la Constitución de la República Federativa del Brasil, en 5 de octubre y que presento en siguiente texto: "É dever do Estado fomentar práticas desportivas formais e não-formais, como direito de cada um". 
En su parágrafo tercero el Artículo 217 determina de manera inequívoca, algunos de los objetivos de la fomentación deportiva en el territorio brasileño: "O Poder Público incentivará o lazer, como forma de promoção social".

Otro hecho de gran relevancia para el universo deportivo en la ciudad de Rio de Janeiro, remite a la extinción de SMEL y por esa razón, la creación de la Secretaria Municipal de Cultura, Turismo y Recreación.

Año de 1993: Regreso de la efectividad y actuación de la Secretaria Municipal de Deporte y Ócio, en la representatividad de la Prefectura de la Ciudad del Rio de Janeiro.

Año de 1996: Implementación de la Villa Olímpica del Salgueiro, proyecto deportivo social que así como la Villa Olímpica de la Mangueira, presentó como característica principal, la ausencia de participación del Gobierno. Esta iniciativa fue implementado en la región norte da la ciudad, en la cerca del "Morro do Salgueiro".

Año de 1997: Inauguración de la Villa Olímpica de la Maré, proyecto deportivo social con gran estructura y administración de la Prefectura de la Ciudad del Rio de Janeiro, pero sólo fue transferido a la atención de la SMEL, en lo año 2000. El respectivo proyecto, fue implementado en una región pobre, acerca de la Bahía de Guanabara, en la zona norte de la ciudad y nombrado como "Complexo da Maré". Es importante señalar que este proyecto sigue el modelo establecido por la Villa Olímpica de la Mangueira, sin embargo, la actuación del gobierno fue incontestable. Cabe señalar, que en la actualidad este proyecto es una referencia para la Prefectura local, además de servir al mayor número de personas entre las villas olímpicas de la ciudad.

En este mismo año, la visita del Presidente de EEUU, Bill Clinton en la Villa Olímpica de la Mangueira, puede haber contribuido para el crecimiento de la visibilidad de los proyectos deportivos sociales, debido la repercusión de este hecho en los medios de comunicación del mundo.

Después de este período, ocurrió una significativa participación del Gobierno de la Ciudad del Rio de Janeiro en la planificación y desenvolvimiento de los proyectos deportivos con finales social. Por lo tanto, entre las muchas formas de proyecto deportivo creados en la ciudad "carioca", las villas olímpicas hay alcanzado gran destaque, siendo implementadas siempre en las localidades pobres de la ciudad.

Año de 2002: Creación de la Villa Olímpica Mestre André, proyecto deportivo social que fue implementado en la comunidad nombrada como "Vila Vintém", en la región oeste de la ciudad.

Año de 2003: Inauguración de la Villa Olímpica Ary Carvalho, en la comunidad nombrada como "Vila Kennedy", en la región oeste de la ciudad y de la Villa Olímpica Clara Nunes, en la comunidad nombrada como "Favela de Acari", en la región norte de la ciudad. Cabe señalar, que la comunidad de la Villa Kennedy, fue una región creada por el Poder Público, durante un proyecto de reorganización habitacional en la ciudad. Año de 2004: Creación de la Villa Olímpica de la Gamboa, en el barrio de la Gamboa, zona portuaria y central de la ciudad. Además, en el mismo año fue creado otro proyecto deportivo social nombrado Villa Olímpica "Oscar Schmidt", en el barrio de "Santa Cruz", en la región oeste de la ciudad.

Año de 2006: Implementación del Proyecto Deportivo Social "Parque das Vizinhanças Dias Gomes", más conocido por la población como "Piscinão de Deodoro", en la región oeste de la ciudad. Cabe señalar, que este proyecto ofrece actividades en el ambiente acuático, además emerge como una significativa posibilidad de entretenimiento para la 
población de la región en los finales de semana y días festivos, ya que sus instalaciones están disponibles para la utilización de la población.

Año de 2007: Entrega del Centro de Entrenamiento Deportivo Miécimo da Silva, que fue inaugurado en lo año de 1982 y está situado en lo barrio de Campo Grande, región oeste de la ciudad. Sin embargo, las instalaciones sufrieran una importante reforma con el fin de recibir las pruebas de los Juegos Panamericanos del Rio de Janeiro en lo año de 2007. Empero, después de las competiciones todas las actividades que se ofrecen al público de la región se reanudaron, con un incremento en el número de puestos disponibles para los participantes, instalaciones nuevas y renovadas.

Año de 2010: Creación de la Villa Olímpica Carlos Castilho, en la comunidad del "Complexo do Alemão", una localidad situada en la región norte de la ciudad y la Villa Olímpica José Manoel Gomes Tubino, proyecto establecido en la comunidad del "Mato Alto", región oeste de la ciudad y que se nombra debido una homenaje para el Baluarte de la Educación Física Brasileña, que falleció en lo ano de 2008.

Año de 2011: Implementación de la Villa Olímpica Artur da Távola, en la comunidad del "Morro dos Macacos", en la zona norte de la ciudad y la Villa Olímpica del Vidigal, proyecto deportivo social situado en la comunidad del "Vidigal", localidad pobre de la región sur de la ciudad y muy conocida debido la visita del Papa João Pablo II, en lo año de 1980.

Año de 2012: Inauguración de la Villa Olímpica Feliz Miéli Venerando, en el barrio de Honório Gurgel, región norte de la ciudad y Villa Olímpica del Caju, iniciativa situada en la "Favela do Caju", región portuaria de la ciudad y que esta acerca de Bahía de Guanabara.

Es importante señalar, que para el año de 2013, la Prefectura de la Ciudad del Rio de Janeiro tiene el planeamiento de la inauguración de mas un proyecto deportivo social, nombrado Villa Olímpica Doutor Sócrates, en el barrio de Pedra de Guarativa, región oeste de la ciudad y que deberá ser nombrada a causa de una homenaje al gran jugador de fútbol brasileño, que falleció en lo año de 2011.

No obstante, es significante deprender, que así como sucede en el proyecto conocido por la población como "Piscinão de Deodoro", todas las villas olímpicas administrados por la SMEL tienen sus instalaciones disponibles para uso del público en los fines de semana y días festivos, un hecho que puede representar un espacio de entretenimiento para la población.

Con respecto a los objetivos asignados en los proyectos deportivos sociales, es posible presentar que la fomentación de la práctica deportiva y la ocupación de tiempo libre de los participantes, son los objetivos principales desde su surgimiento, debido la necesidad de la reducción de las posibilidades del envolvimiento de los jóvenes con las situaciones y acontecimientos que pueden expresar riesgo social, tales como la violencia, la delincuencia y el consumo de drogas. Este objetivo se basa en los principios y el potencial del deporte, a través de la diseminación de los preceptos de valor positivo.

Sin embargo, en 2008 la SMEL, establece los principales objetivos y metas de los proyectos deportivos sociales, dirigiendo y reglando el servicio en todas las unidades de su administración, siendo presentado la aplicación de las siguientes acciones: evaluar la incidencia de infracciones, reducir la evasión escolar y promover la inclusión social de las personas con discapacidad. 
Por otra parte, los proyectos tienen la obligación de promover la actividad económica en la región, el aumento de la expectativa de vida de la tercera edad, reducir el número de acontecimientos relacionados con problemas respiratorios, democratizar el acceso al entretenimiento, promover el entendimiento de los valores familiares y el descubrimiento de talentos deportivos. Siendo así, es esperado obtener resultados tales como la detección y orientación de los atletas deportivos, la creación de empleo y de rendimiento económico para el público de la región, la evaluación de la incidencia de las infracciones, la integración entre las comunidades, la vigilancia epidemiológica, la promoción de la salud, la promoción de la auto-autoestima, mejorar el rendimiento académico y reducir la evasión escolar, asistiendo así una gran variedad de posibilidades que se pueden ofrecer a través del desenvolvimiento del deporte.

\section{4- DISCUSIÓN}

Los resultados del estudio indicaron una preocupación del gobierno en favor de la diseminación deportiva, principalmente entre los sectores más necesitados de población y que en su mayoría presentan ambiente de degradación social, como presentado por Vargas (2002). Aunque, Maricato (2001) y Souza (2009) infieren que estas localidades, han surgido en la sociedad brasileña, debido la ausencia o insuficiencia de la acción pública, hecho que provocó el desarrollo de una brecha que llevó a la creación de regiones con características de degradación social y de barrios marginales, además de padecieren con la falta de infraestructura y servicios sociales.

De acuerdo con Morais (2006) las comunidades pobres tienen escenario peculiar, expresando acontecimiento y ambiente con características perjudiciales para el público joven. Ferreira (2006) afirma que tenemos que cuidar de lo ambiente de inserción de los jóvenes, ya que el contexto social con característica de abandono y pobreza exponen el público a situaciones clasificadas como de riesgo social, siendo necesarias acciones del gobierno para evitar que esto ocurra, como determinado entre los objetivos de la SMEL.

Entre los esfuerzos de lo gobierno brasileño para la creación de dispositivos legales que tienen como objetivo reducir o combatir los problemas sociales producidos en la sociedad contemporánea, se ha convertido en posible citar el Artículo 217 de la Constitución de la República Federativa del Brasil, promulgada el 5 de octubre de 1988, en relación a fomentación del deporte en su diferentes dimensiones sociales, hecho que favoreció para el implemento del deporte como una estrategia de entrenamiento de las habilidades sociales y fue presentado entre los objetivos de las villas olímpicas de la ciudad del Rio de Janeiro, través del Manual das Vilas Olímpicas da Prefeitura do Rio de Janeiro (2008).

La promulgación del Artículo 217 de la Constitución de La República del Brasil, en 1988, en lo caso especifico de la ciudad del Rio de Janeiro, emerge como un hecho significante, donde la Prefectura local, después de esta fecha inicia la diseminación deportiva con gran destaque, principalmente tiendo los proyectos deportivos sociales como estrategia principal, que en este estudio fueran representados por las villas olímpicas. Es importante el entendimiento que en la actualidad esta determinación del gobierno del Rio de Janeiro, continúan con crecimiento significativo, principalmente en el atendimiento de la población pobre.

Sá (1999) señaló que la combinación de un público joven con el ambiente pobre, ofrece mayor probabilidad de ocurrencia de una postura y el comportamiento agresivo, situación que también se ha presentado en el estudio de Pavarino, Prette y Prette (2005). Según Tubino (2001) y Melo (2004), el deporte se ha establecido en la 
sociedad actual, especialmente por combatir los problemas sociales, tiendo resultados positivos en el entrenamiento de las habilidades sociales, surgiendo como una alternativa, especialmente para personas pobres. Tales argumentos ratifican la postura adoptada por la Prefectura de la Ciudad del Rio de Janeiro, en relación a la difusión de los proyectos deportivos sociales, estableciendo como objetivo prioritario la formación de habilidades sociales y en consecuencia la oferta de ayuda a la población, especialmente los pobres. (Secretaria Municipal de Esporte e Lazer, 2008).

Los proyectos deportivos sociales, junto con las metas establecidas por la SMEL, ganaran notoriedad principalmente en los argumentos de autores como Alves y Pieranti (2007) que informaron la necesidad de una política con el objetivo de desarrollo del deporte en la República Federativa del Brasil, siendo este un derecho indubitable de los ciudadanos brasileños, en conformidad con el Artículo 217 de la Constitución de la República Federativa del Brasil, promulgada el 5 de octubre de 1988 y hoy es parte del Manual das Vilas Olímpicas da Prefeitura do Rio de Janeiro (2008).

Hillesheim y Cruz (2008) en conformidad con Contijo y Medeiros (2009), destacaran la importancia de entendimiento y análisis del ambiente de inserción del individuo, dada la influencia que el contexto social puede expresar, sobre todo para el público joven que está en proceso formación de la identidad. Bronfenbrenner (1992), Krebs (1997) y Vargas (2002) mostraron que el contexto social asume una función clave en la formación de un individuo, que pueden expresar características positivas y negativas presentes y experimentadas a lo largo de su vida. Por lo tanto, los proyectos deportivos sociales, villas olímpicas, a través de actividades deportivas y de difusión de evaluación preceptos positivos, contribuyen positivamente a competir por estos individuos, principalmente por estar situados en localidades donde la influencia no es buenas para el desenvolvimiento de las personas.

No obstante, Vargas (2002) presentó que en el caso específico de los niños, la presentación de la motricidad con estereotipia degradada es una característica de gran importancia, ya que este hecho es una consecuencia de la exposición a situaciones degradantes y que muchas veces estos jóvenes acaban por reproducir. Cabe señalar que estas situaciones están presentes en el contexto de las comunidades pobres como enfocarán Dias (1996) y Neto (2001).

Andrade (1998) y Scherer-Warren (2006) deprenderán que diversas iniciativas se desarrollan en la sociedad brasileña, con el objetivo de reducir las posibilidades contacto de los niños con las situaciones que presentan las características de riesgo social, presentando así una idea oposición a enunciada ocurrencia, sobre todo con la reducción de tiempo libre de estos individuos, como fue presentado entre los objetivos de los proyectos gestionados por la SMEL.

Silveira (2006) y Correia (2008) mostraron que la ejecución de los proyectos sociales que tienen una relación directa con el deporte consolidaron su efectividad en la sociedad contemporánea. Además, su designación y sus resultados son analizados científicamente, como hacen los autores como Rezende (2002), Dória y Tubino (2006), donde sus investigaciones presentaran supuestos beneficios proporcionados por un determinado proyecto deportivo social, nombrado Villa Olímpica de la "Mangueira". Este tema es muy importante, siendo posible encontrar otros estudios en la literatura como la posible consolidación de este tipo de política en la sociedad contemporánea, que en ese momento apareció como estrategia importante y válida para el público en situación de riesgo y exclusión social, especialmente de los niños. 
Hay que señalar que este tipo de iniciativas creció en escenario de la ciudad de Río de Janeiro, lo que favorece la consolidación y difusión de la política deportiva social, que hoy es fomentada principalmente por el gobierno, según lo indicado por Machado, Doria y Vargas (2011) y puede determinarse durante el estudio.

\section{5.- CONCLUSIONES}

Destarte, fue posible llegar al entendimiento que el crecimiento de los proyectos, así como la valorización del deporte en la perspectiva social, ocurrió después de la promulgación del artículo 217 de la Constitución de la República Federativa del Brasil, 5 de octubre de 1988.

Los resultados de la investigación permiten concluir que el crecimiento de los proyectos deportivos sociales y por lo tanto, la valorización del deporte en la ciudad de Rio de Janeiro, presentaran un importante desenvolvimiento después de la participación del gobierno, sin embargo, no podemos dejar de destacar que esta iniciativa nació debido una acción que no ha recibido el apoyo del gobierno.

Otro factor que hay corroborado para el crecimiento de la diseminación deportiva con fines sociales, respecta el aumento de los problemas sociales presentes en la sociedad contemporánea, especialmente en la ciudad de Río de Janeiro, que sufre con los efectos y con la influencia de este tipo de problemas, que en su mayoría hacen como victima la población joven y tienen un presencia muy grande entre las camadas más bajas de la población.

La estandarización y direccionamiento del servicio en proyectos deportivos sociales llamado Villa Olímpica, junto con el establecimiento de metas y objetivos a alcanzar, reforzó el escenario de visibilidad asumido por del deporte en la sociedad contemporánea, especialmente en lo que se refiere a la formación de las habilidades sociales, objetivo siempre deseado en los proyectos de esta magnitud y de la gran importancia para la sociedad.

El escenario actual permite decir que en la ciudad de Río de Janeiro las villas olímpicas son una realidad y también presentan tendencia de crecimiento significativo debido a los beneficios y amplitud de trabajo que se presenta en este tipo de proyectos. Por lo tanto, la consolidación del deporte, como estrategia social se convirtió en indiscutible en la sociedad contemporánea y sus objetivos apuntan a satisfacer los anhelos de la población, que en su mayoría provienen de los sectores más desfavorecidos de la sociedad.

\section{6.- BIBLIOGRAFÍA}

Acosta-Cázares, B, Aranda-Álvarez, J, Reyes-Morales, H (2006) ENCOPREVENIMSS 2004 Patrones de actividad física de la mujer y del hombre. Revista Médica del Instituto Mexicano del Seguro Social, 44 (11): 79-86.

Ainsworth, BE, Haskell WL, Leon, AL, Jacobs, DR, Montoye, H.J, Sallis, JF, Paffenbarger, RS (1993). Compendium of physical activities: classification of energy costs of human physical activities. Med. Sci. Sports Exerc. 25: 71-80.

CENTER FOR DISEASE CONTROL AND PREVENTION (1996) Neighborhood Safety and the Prevalence of Physical Inactivity-Selected Status. Journal of the American Medical Association, 281, 15. 
COMISIÓN NACIONAL DEL DEPORTE (2001) Programa Nacional de Cultura Física y Deporte 2001-2006, México. (En línea). http://www.conade.gob.mx/paginas_07/doc_basicos.asp.

Craig, CL, Marshall, AL., Sjostrom, M, Bauman, AE, Booth, ML, Ainsworth, BE (2003) International physical activity questionnaire: 12-country reliability and validity. Medicine and Science in Sports and Exercise, 35: 1381-95.

Kannell, WB, Sorlie, P (1979) Some health benefits of physical activity. The Framingham Study. Archives of Internal Medicine, 139: 857 - 861.

ORGANIZACIÓN PANAMERICANA DE LA SALUD (2003) Consulta Regional OPS sobre Alimentación Saludable y Actividad Física en las Américas. (En línea). http://www.dpaslac.org/uploads/1154103060.pdf.

Paffenbarger, R, Hyde, R, Wing, A, Lee, I, Jung, D, Kampter, J (1993) The association of changes in physical activity level and other lifestyle characteristics with mortality among men. New England Journal of Medicine, 328: 538-545.

Peña, M, Bacallao, J (2001) "La obesidad y sus tendencias en la región". Revista Panamericana Salud Publica, 10 (2): 75.

Rennie, KL, McCarthy, N, Yazdgerdi, S, Marmot, M, Brunner, E (2003) Association of the metabolic syndrome with both vigorous and moderate physical activity. International Journal of Epidemiology, 32: 600-606.

Rivera-Dommarco, J, Cuevas, L, Shamah-Levy, T, Villalpando, S, Avila, M, Jiménez, A (2006) Encuesta Nacional de Salud y Nutrición 2006. (Estado Nutricio) (En línea). http://www.insp.mx/ensanut/ensanut2006.pdf (consulta: 22 agosto de 2008).

Wingard, DL (1982) The sex differential in mortality rates: demographic and behavioral factors. American Journal of Epidemiology, 115: 205-216. 